\title{
The type I Lanyu pig has a maternal genetic lineage distinct from Asian and European pigs
}

\author{
C. Y. Wu*, $\mathrm{Wu}^{*}$, Y. N. Jiang ${ }^{*, 1}$, H. P. Chu ${ }^{\dagger}$, S. H. $\mathrm{Li}^{\ddagger}$, Y. Wang ${ }^{\ddagger}$, Y. H. Li ${ }^{*}$, Y. Chang ${ }^{\S}$ and Y. T. Ju* \\ *Department of Animal Science and Technology, National Taiwan University, Taipei 10673, Taiwan. ${ }^{\dagger}$ Taitung Animal Propagation Station, \\ Livestock Research Institute, Taitung 95444, Taiwan. ${ }^{\ddagger}$ Department of Life Science, National Taiwan Normal University, Taipei 11677, \\ Taiwan. ${ }^{\S}$ Council of Agriculture, Executive Yuan, Taipei 10014, Taiwan
}

\section{Summary}

\begin{abstract}
The Lanyu pig is an indigenous breed from Lanyu Islet, located south-east of Taiwan, with phenotypic characteristics distinctive from other pig breeds in Asia and Europe. Based on geographic considerations, the Lanyu pig may have originated from mainland China, Austronesia or the Ryukyu Islands. In the present study, polymorphism of the mitochondrial DNA control region sequence was used to clarify phylogenetic relationships among two herds of Lanyu pigs imported before 1980 from Lanyu Islet into Taiwan and reared in isolation on two different farms. Two distinct mitochondrial control region haplotypes were found. The type I Lanyu sequence appeared independently as a unique clade different from Asian and European pig sequences, while the type II Lanyu sequence was clustered within the major Asian clade. The pairwise distances between the major Asian clade vs. the type I Lanyu and European clades were $0.01726 \pm 0.00275$ and $0.01975 \pm 0.00212$ changes per site respectively. Estimates of divergence time suggest that the type I Lanyu sequence split from the major Asian pig clade in prehistoric times. The type II Lanyu mtDNA shares a close genetic lineage with Japanese Satsuma and New Zealand Kune Kune mtDNA with pairwise distances of $0.00095 \pm 0.00000$ and $0.00192 \pm 0.00000$ respectively, indicating gene flow between Lanyu Islet, Japan and Oceania in recent times. Together these results indicate that the type I Lanyu pig has a genetic lineage separate from Asian-type pigs, while the type II Lanyu sequence may represent a more recent introgression of modern Asian pigs.
\end{abstract}

Keywords control region, genetic diversity, Lanyu pig, mitochondrial DNA, phylogeny, polymorphism.

\section{Introduction}

The Lanyu pig is an indigenous pig breed from Lanyu (Orchid) Islet off the south-east coast of Taiwan. These pigs possess a narrow head, long straight snout, small erect ears and coarse dark hairs. The adult body weight is about $70 \mathrm{~kg}$ and is categorized as a miniature pig (Fig. 1a). The Lanyu pig differs from other breeds distributed in southern China, which possess bigger ears and a larger body (Cheng 1986). Lanyu pigs might share genetic lineage with pigs from mainland China, Austronesia, Oceania and Japan based on geographic proximities (Fig. 1b).

\section{Address for correspondence}

Y. T. Ju, Department of Animal Science and Technology, National Taiwan University, Taipei 10673, Taiwan.

E-mail: ytju@ntu.edu.tw

${ }^{1}$ Both of these authors contributed equally to this work.

Accepted for publication 27 June 2007
To develop a laboratory pig stock, two herds of indigenous Lanyu pigs with black coat colour were moved from Lanyu Islet to Taiwan. One herd (one male and three females) was placed on the teaching farm of National Taiwan University (NTU) in 1975 and another went to the Taitung Animal Propagation Station (TAPS) in 1980 (four males and sixteen females). These pigs were reared in isolation as two separate herds, and randomly mated until 2004. The genetic diversity and phylogenetics of the two herds have not yet been characterized.

Many exotic pig breeds were introduced into Taiwan by early colonizers. The Taoyuan pig breed was introduced from mainland China into Taiwan following extensive southward migration of colonizers at the end of the 19th century (1877-1887) (Cheng 1986). The Japanese imported the Berkshire breed to improve the performance of Taoyuan pigs when they occupied Taiwan between 1895 and 1945. The Yorkshire, Hampshire, Landrace and Duroc pig breeds were introduced into Taiwan between 1948 and 

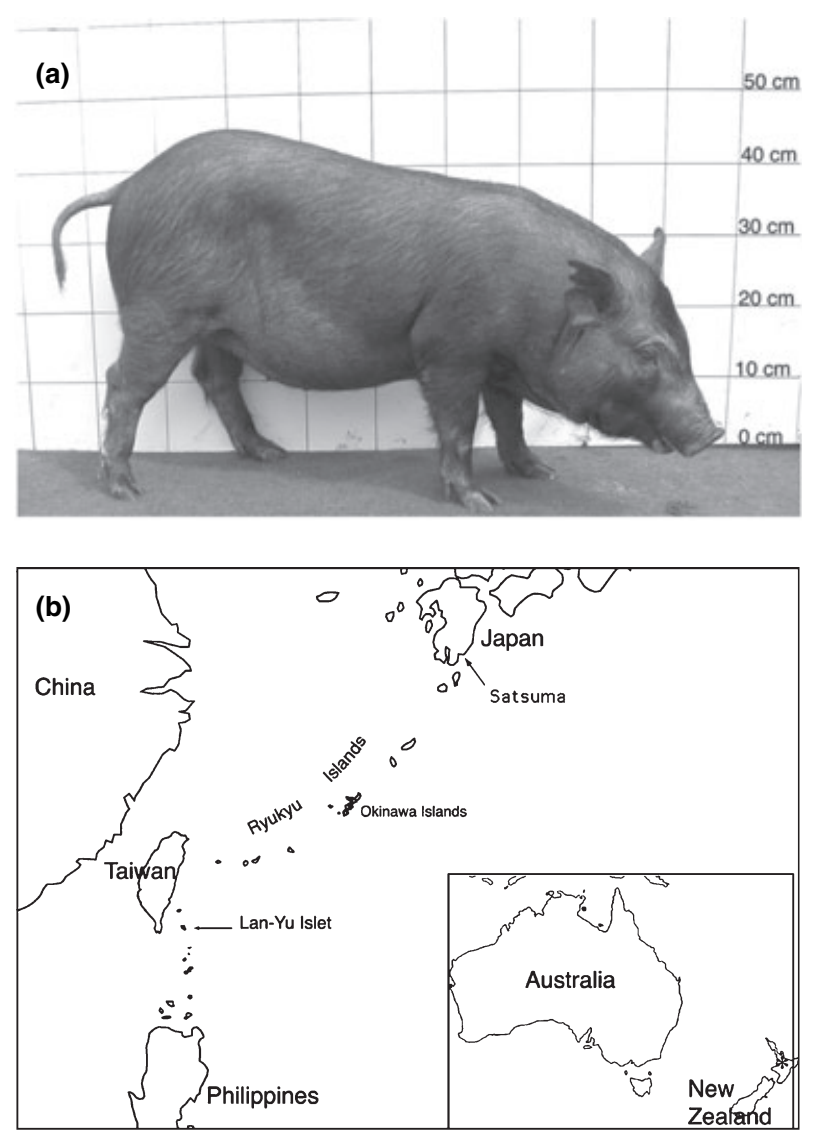

Figure 1 (a) A mature 5-month-old female Lanyu pig. (b) Map of Taiwan, Lanyu Islet, mainland China and the Ryukyu and Philippine archipelagos. The inset box shows continental Australia and New Zealand, where an asterisk $\left(^{*}\right)$ indicates the location of the Kune Kune pig from New Zealand.

1974. Genetic introgression into the Lanyu breed may have occurred following the introduction of these exotic pig breeds.

Mitochondrial control region sequence polymorphism is commonly used to explore intraspecies evolution and divergence (Saunders \& Edwards 2000; Wan et al. 2004) and has previously been used to study the phylogenetic relationships of Asian and European pig breeds and Australian and New Zealand feral pigs (Kim et al. 2002; Watanobe et al. 2002, 2003; Gongora et al. 2004). To explore the origin of the Lanyu pigs and their phylogenetic relationships among Asian and European pigs, the mitochondrial DNA control regions of conserved Lanyu pigs were sequenced and compared with previously determined control region sequences of Asian and European pigs.

\section{Materials and methods}

Sample collection and preparation of mitochondrial DNA

Blood samples were collected from 44 heads of Lanyu pigs from the isolated herds at TAPS (39 animals) and NTU teaching farm (five animals). Mitochondrial DNA was extracted and purified from platelet-rich plasma using Qiagen's QIAamp DNA mini kit. The quality of purified mtDNA was analysed in $1 \%$ agarose gel by electrophoresis.

Design of primer sequences and PCR amplification of mtDNA control region

The entire mtDNA control regions were amplified by PCR in an MJ thermocycler using the following primers: L1 (5'-CCAAGACTCAAGGAAGGAGA-3'; nucleotide positions 16542-16561 of pig mtDNA sequence AF034253) and H1 (5'-GGCGCGGATACTTGCATGTG-3'; positions 12901309). After standard PCR amplification, the control regions were sequenced in both directions using the L1 and H1 primers, as well as the following: L2 (5'-CCTATGTACGTCGTGCATTA-3'; positions 160-179), L3 (5'-TACTTCAGGACCATCTCACC-3'; positions 434-453), H2 (5'-AGTGTAAGTTAGGCTTATTG-3'; positions 963-982) and H3 (5'-TTGTGGTAGATTGGCGTAAA-3'; positions 1072-1091). Full sequences of the control region were assembled from overlapping forward and reverse sequences with SEOEDIT software (DNASTAR Inc.; Hein \& Stovlbaek 1996). The full length of type I Lanyu (EF375877) and type II Lanyu (DQ972936) pig mtDNA sequences have been deposited into GenBank. Other pig sequences obtained from GenBank are listed in Fig. 2.

\section{Data analysis}

Control regions from 43 pig breeds previously published by Okumura et al. (1996), Kim et al. (2002), Gongora et al. (2004) and Watanobe et al. (2002), as well as Chinese and Asian sequences deposited in GenBank (Fig. 2) were aligned with the 44 Lanyu pig sequences obtained in this study. The common highly variable tandem repeat motif 5'-CGTGCGTACA-3' and the specific repeat sequence motifs 5'-ACACAAACC-3' and 5'-TAAAACACTTA-3' in Lanyu mtDNA control regions were excluded from the analysis. Multiple alignment of pig sequences was performed using MEGALIGN software (DNASTAR Inc.). Pairwise distances of individual sequences were measured using DNA Sequence Polymorphism (DNASP) software (Rozas et al. 2003). PHYLIP version 3.6 was used to generate the phylogenetic tree by maximum likelihood (ML) methods (Felsenstein 1993, 2006). For ML analysis, we used software MODELTEST version 3.6 (Posada \& Crandall 1998) to determine the best fit model of the data, including nucleotide composition, substitution matrix among nucleotides, proportion of invariant sites and $\Gamma$ shape distribution parameter $(\alpha)$. Nodal supports of the ML tree were evaluated by bootstrap resampling (1000 replications) using the fast heuristic search algorithm implemented in software PAUP4.0 $\beta 10$ (Swofford 2002). TREEPUZZLE version 5.0 software (based on a quartet puzzling method) was used to calculate confidence numbers (puzzle 

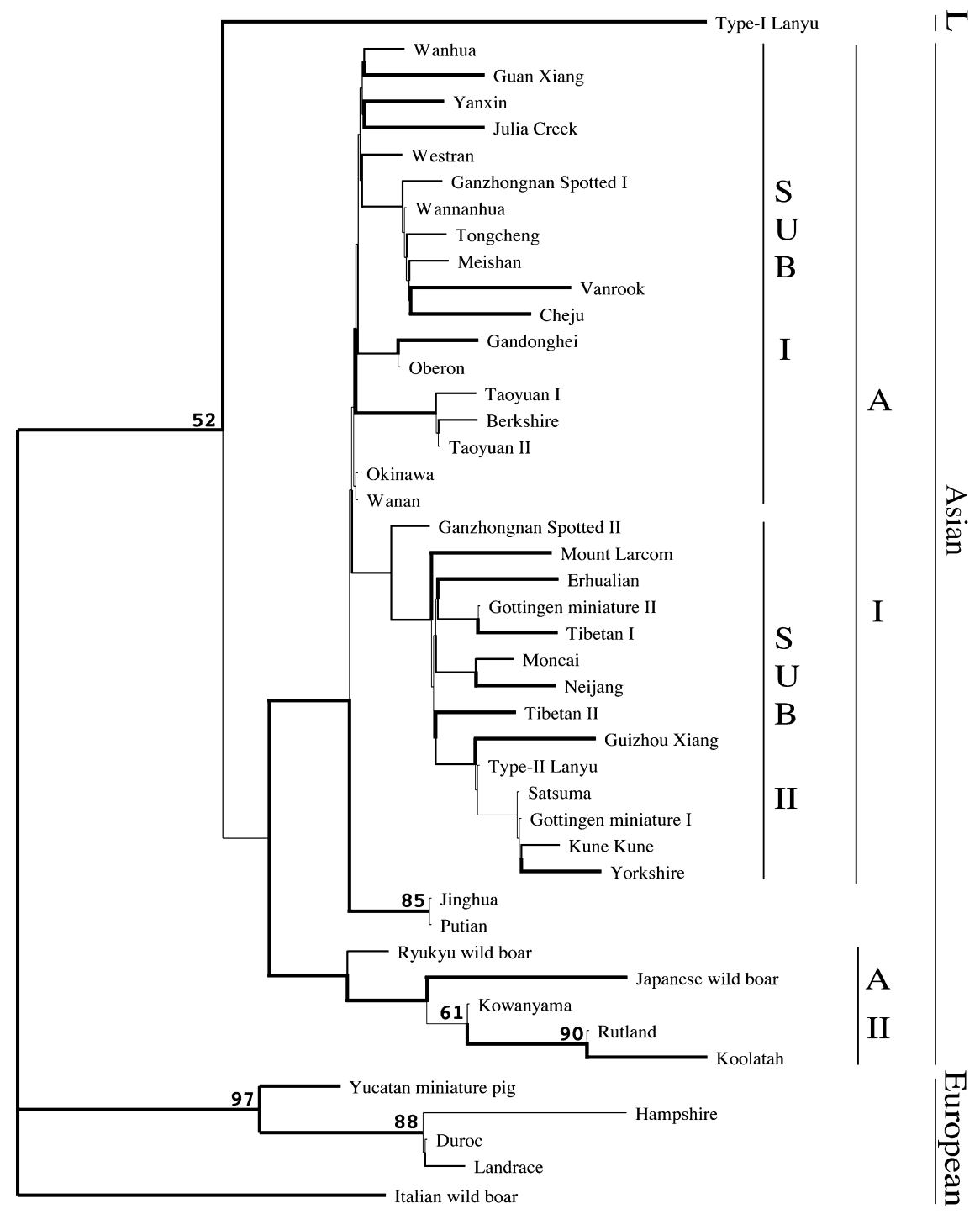

0.01

Figure 2 Maximum likelihood phylogenetic tree of the Lanyu, Taoyuan, Asian and European pig sequences. Sequences include type I Lanyu (EF375877), type II Lanyu (DQ972936), Taoyuan I (AM040645), Taoyuan II (AM040646), Okinawa native boar (AB015092), Wan'an (AF276924), Oberon (AY463088), Westran (AF276921), Wanhua (AF276932), Yanxin (AF276927), Berkshire (AM040639), Ganzhongnan Spotted I (AY463061), Ganzhongnan Spotted II (AY486115), Tongcheng (AF276923), Wannanhua (AF276925), Meishan (D17739), Putian (AF276931), Jinghua (AF276930), Gandonghei (AF276928), Julia Creek (AY463092), Guan Xiang (AY486117), Neijang (AF276929), Moncai (AB041481), Tibetan I (AY463062), Guizhou Xiang (AY486118), Tibetan II (AY486116), Satsuma (AB015091), Göttingen miniature pig I (AY463068), Yorkshire (AM040635), Göttingen miniature pig II (AY463067), Kune Kune (AY463076), Mount Larcom (AY463093), Erhualian (AF276922), Cheju (AF276933), Vanrook (AY463094), Koolatah (AY463096), Rutland (AY463095), Japanese wild boar (AB015085), Ryukyu wild boar (AB015087), Kowanyama (AY463091), Hampshire (AY429460), Yucatan miniature pig (AB015093), Landrace (AM040615), Duroc (AM040625) and Italian wild boar (AB015094). Highly significant and significant confidences of branches are shown by bold and middle-weight lines respectively. Numbers on the branches are bootstrap values based on bootstrap resampling (1000 replications).

support values) in the phylogeny and likelihood-mapping analyses (Schmidt et al. 2002). The significance of any difference among pig groups was tested using 10000 permutations in the quartet puzzling algorithm (Strimmer \& von Haeseler 1996). The molecular clock equation $T=$ $K / 2 r$ was used to estimate the time since divergence $(T)$, with substitution rates $r=1.5 \times 10^{-7}$ and $1 \times 10^{-8}$ substitutions/site/year and sequence divergence $(K)$ from the results above (Bradley et al. 1996; Giuffra et al. 2000; Kim et al. 2002). The upper and lower divergence times were estimated assuming that the corrected nucleotide difference between European and Asian Sus scrofa (0.01976) 
accumulated for 58000 (Kim et al. 2002) or 500000 (Giuffra et al. 2000) years before present (YBP).

\section{Results}

Variation of mtDNA control region sequences among Lanyu, Asian and European pigs

To investigate the phylogenetic relationship among Lanyu, Asian and European pigs, mtDNA control region sequences were obtained from 44 conserved Lanyu pigs, 19 indigenous pigs (including two Taoyuan pigs) from mainland China, one pig from Vietnam, four feral pigs from Japan, a Cheju pig from Korea, nine feral pigs from Australia and New Zealand, two Göttingen mini pigs, six domestic pigs and a wild boar from Europe. Of the feral pigs distributed in Australia and New Zealand, only Asian-type feral pigs were included in this study (Gongora et al. 2004). After comparison of polymorphisms of the mtDNA control region, only two control region haplotypes (referred to as type I and II Lanyu) were identified in the conserved Lanyu pigs, indicating a serious lack of heterozygosity in the population (Fig. S1). Lanyu pigs reared in TAPS had a mixture of type I (28 animals) and type II (11 animals) mtDNA haplotypes, while all NTU teaching farm pigs had the type I haplotype. The phenotypic characteristics of the Lanyu pigs in the two herds were indistinguishable.

One hundred and twenty-nine polymorphic sites in the mtDNA control region were identified, comprising 121 transitions, eight transversions and one single-nucleotide deletion/insertion (Fig. S2). A triplicate of the ACACAAACC (positions 998-1006; Fig. S1) motif was specifically found in pigs with type I Lanyu mtDNA, while just one ACACAAACC motif was found in other sequences used in this study. All type II Lanyu and Japanese Satsuma pig sequences shared another duplicated motif (TAAAACACTTA, positions 1018-1028; Fig. S1). This motif was present only once in other sequences used in this study. As a result, the length of the mtDNA control region in both Lanyu haplotypes varied due to absolute differences in the numbers of $5^{\prime}$-CGTGCGTACA- $3^{\prime}$, 5'-ACACAAACC-3' and 5'-TAAAACACTTA-3' motifs present in their control regions.

Within all of the conserved Lanyu pig sequences, 17 transitions and one transversion were found in the mtDNA control region. The nucleotides in positions 109, 131, 145, 390 and 1089, which are diagnostic between Asian and European pigs (Gongora et al. 2004), were found consistently in both Lanyu sequences to be Asian pig-specific nucleotides, indicating a close genetic affinity of Lanyu pigs to Asian pigs (Fig. S2). Additionally, transitions in nucleotide positions 302, 391, 535, 542 and 657 , and a transversion at position 871 (adenosine in consensus sequence and thymine in type I Lanyu) were found in the control region of type I Lanyu mtDNA but not in other sequences used in this study.

\section{Phylogenetic relationship of Lanyu, Asian and European pigs}

To compare the genetic lineages of Lanyu, Asian and European pigs, the pairwise genetic distance among their control regions was determined and ML phylogenetic tree was constructed. The most appropriate model for this data set was found to be TIM $+I+\Gamma(-\ln =2202.8789 ; K=$ $8 ; \mathrm{AIC}=4421.7578)$. The ML estimates of base frequencies were as follows: A, 0.3622; C, 0.2636; G, 0.1229 and T, 0.2513 . Estimated symmetrical substitution rates among these nucleotides were 1.0000 for $\mathrm{A} / \mathrm{C}, 460.2917$ for $\mathrm{A} / \mathrm{G}$, 253.1900 for $\mathrm{T} / \mathrm{C}, 18.3488$ for $\mathrm{A} / \mathrm{T}, 18.3488$ for $\mathrm{C} / \mathrm{G}$ and 1.0000 for G/T. The estimated shape parameter $(\alpha)$ for the $\Gamma$ distribution was 0.7261 . The estimated proportion of invariable sites was 0.7723 . A transition/transversion ratio of $18.44 \%$ was used to obtain the ML tree, which contained three major clades (Fig. 2). The treelike topology and phylogenetic signal obtained by the quartet puzzling method (quartet puzzling support value, $71.3 \%$ ) supported the branch assignments in this phylogenetic tree. The European pig sequences (excluding Berkshire and Yorkshire breeds) clustered together to form one major clade (European clade, 97\% bootstrap value), separated from the major Asian clade (Fig. 2). The type I Lanyu sequence independently clustered as a unique clade (L clade) with $52 \%$ bootstrap value, while the type II Lanyu sequence fell into the main Asian clade. Two separate subclades within the Asian clade could also be recognized: one subclade (AII) included mtDNA from the Ryukyu wild boar, Japanese wild boar, Kowanyama, Rutland and Koolatah pigs. The remaining Asian pigs, type II Lanyu, New Zealand and other Australian feral pig sequences were clustered together within the other subclade, AI. Berkshire and Yorkshire sequences fell in the AI subclade, within the Asian clade; this result was consistent with earlier analyses (Kim et al. 2002; Gongora et al. 2004). The AI subclade could be further divided into Sub I and Sub II divisions (Fig. 2). The Jinghua and Putian sequences lay separately within the Asian clade, with $85 \%$ bootstrap value.

Average pairwise difference between Asian and European clades was $0.01975 \pm 0.00212$ changes per site. The mean pairwise distance among Asian sequences was $0.00607 \pm 0.00059$. These data are in agreement with Watanobe et al. (2003). Pairwise distances between type I Lanyu clade vs. Asian and European clades were $0.01726 \pm 0.00275$ and $0.02216 \pm 0.00889$ respectively. The time since divergence of Asian and European mtDNA sequenes was calculated to be 32 917-493 750 YBP. The divergence time of type I Lanyu clade and Asian clade was 28 767-431 500 YBP. The type II Lanyu sequence was closely aligned with Satsuma, Göttingen, Kune Kune and 
Yorkshire sequences (pairwise difference: $0.00095 \pm$ $0.00000 ; 0.00144 \pm 0.00083 ; 0.00192 \pm 0.00000$ and $0.00288 \pm 0.00000$ respectively). A close genetic affinity was identified between Taoyuan and Berkshire sequences, with a pairwise distance of $0.00144 \pm 0.00083$.

\section{Discussion}

\section{The origin of Lanyu pigs}

In this study, only two mtDNA control region haplotypes were found in the conserved Lanyu pigs. When compared with Asian and European type pigs, the type I Lanyu mtDNA control region not only possesses a unique triplicate ACACAAACC motif, but also contains five unique transitions (in positions 302, 391, 535, 542 and 657) and one unique transversion (position 871). Thus, the type I Lanyu animals have a distinct genetic lineage from Asian and European pigs, indicating that the Lanyu pig breed might have been formed in pre-history. However, the type I Lanyu sequence shares specific characteristic nucleotide substitutions with Asian pig mtDNA control region sequences (Gongora et al. 2004), indicating that the type I Lanyu sequence is closely related to Asian pigs. Based on these two points, two hypothetical situations for the origin of type I Lanyu may have occurred. Either the original Lanyu pig shares a common ancestor with Asian pig breeds or the Lanyu pig has an origin distinct from all known Asian pigs.

The Ryukyu archipelago lies just north-east of Taiwan. The Ryukyu wild boar has a distinct genetic lineage from Asian pigs, based on comparisons with the mtDNA control region of modern Ryukyu and Japanese wild boars, Asian pigs and ancient Ryukyu wild boars from mtDNA of bone specimens excavated from archaeological sites on Okinawa islands (Watanobe et al. 1999, 2002, 2003). The genetic divergence between Ryukyu wild boar and Asian pigs' clade has been estimated as $0.00727 \pm 0.00123$. Nevertheless, the pairwise distance between type I Lanyu sequence and the major Asian AI clade (0.01731 \pm 0.00304 ) is greater than the Ryukyu wild boar vs. major Asian AI clade, and the pairwise distance between type I Lanyu and Ryukyu wild boar is $0.01548 \pm 0.00000$. This result suggests that the formation of the original Lanyu pig and the Ryukyu wild boar might be the result of distinct historical events.

The time of entry of modern humans into eastern Asian from mainland Southeast Asia has been estimated at about $60000 \mathrm{YBP}$, coinciding with the receding of continental glaciers (Su et al. 1999, 2000) around 15 00075000 years ago (Dawson 1992). Here, we identified that the type I Lanyu sequence split from Asian sequences some 28 767-431 500 years ago, and is a unique mtDNA quite different from modern Asian pigs. From these results, we hypothesize that the formation of a unique Lanyu pig breed, identified by the type I Lanyu sequence, might be the result of biogeographical isolation following the last continental glacier recession, and that the mtDNA did not introgress into pigs distributed in mainland China and north Asia by prehistoric human migrations.

\section{Genetic introgression of type II Lanyu from Asian pigs}

The type II Lanyu mtDNA sequence clustered within the major Asian pig clade and is closely related to sequences from the Japanese domestic pig (Satsuma), the Kune Kune, the Yorkshire and two Göttingen miniature pigs, although with low bootstrap values. Interestingly, an identical duplicate TAAAACACTAA motif of the mtDNA control region is found in both type II Lanyu and Satsuma sequences. Only a single transition (position 241, thymine in type II Lanyu and cytosine in Satsuma) was found in the entire control region of these two sequences. This strongly suggests both pigs' mtDNA have the same origin. The Satsuma pig is a modern pig breed distributed on mainland Japan, with a typical Berkshire phenotype. Its genetic lineage is different from Ryukyu and Japanese wild boars, according to a molecular study (Okumura et al. 1996). Although the Ryukyu Islands are directly between Taiwan and mainland Japan, the specific type II Lanyu and Satsuma mtDNA haplotypes are absent from the Ryukyu Islands, suggesting that the observed gene flow might have arisen through human migration or trade. Pig farming in mainland Japan was interrupted from eighth century to the late 19th century by Buddhist prohibition (Watanobe et al. 2002). When the Japanese governed Taiwan from 1895 to 1945 , they introduced pigs from Taiwan to improve domestic pig stocks. In the light of this history, these results suggest that the type II Lanyu sequence was introgressed from nearby islands into the Satsuma pig of mainland Japan in recent times.

The Kune Kune, Göttingen miniature, Satsuma and Yorkshire sequences were classified together with the type II Lanyu sequence, consistent with previous findings (Gongora et al. 2004). The Göttingen miniature pig was created by crossing Minnesota miniature pigs and small Vietnamese potbelly pigs (Bollen et al. 2000). As Taiwan and Lanyu Islet are located in the intersection of mainland Asia and Kuroshio Current, the close genetic similarity among these pigs probably indicates gene flow resulting from recent human migration. We believe that the type II Lanyu haplotype represents introgression from pig breeds from south Asia into the original Lanyu pig.

The Lanyu and Taoyuan pigs possess different mtDNA haplotypes

The Taoyuan pigs clustered with nine Chinese pig breeds, one Japanese native pig (Okinawa), one Korean pig (Cheju pig), four Asian-type Australian feral pigs (Julia Creek, Oberon, Vanrook and Westran pigs) and one Chinese pig breed of European descent, the Berkshire. In terms of 
mtDNA control region genetic distance, the Taoyuan pig is very close to the Berkshire breed. The Berkshire was the first exotic pig breed introduced into Taiwan by Japanese in 1898. Together with our finding, this history suggests introgression of Berkshire genes into the Taoyuan pig breed during the Japanese colonial period in Taiwan. The pairwise distance and divergence time between type II Lanyu sequence (Sub II clade) and Taoyuan sequence (Sub I clade) are $0.00529 \pm 0.00268$ and 8817-132 250 YBP respectively, indicating that the two breeds have very different lineages.

\section{Lanyu pig chromosomal diversity}

As the mitochondrial DNA is a cytoplasmic genome and is maternally inherited, its replication mechanism and evolution do not reflect the history of the nuclear genome. A preliminary survey was undertaken to understand the relationship of mtDNA haplotypes to nuclear DNA diversity in the conserved Lanyu pigs. Nuclear genotyping of all conserved Lanyu pigs was performed using 19 microsatellite primers, randomly located on each chromosome, following a scheme proposed under the FAO Domestic Animal Diversity Information (FAO 2004). This phylogenetic analysis showed that the microsatellite genotypes of conserved Lanyu pigs were subdivided into four populations, and pigs with the two different haplotypes of mtDNA were randomly distributed within these four sub-populations (data not shown). Future analyses of the phylogenetics of the Lanyu pigs will require both mtDNA and nuclear DNA profiling.

In summary, we have shown that Lanyu pigs possess only two haplotypes of mtDNA. The type I haplotype is unique to Lanyu pigs, and suggests that the original Lanyu pig has a maternal genetic lineage distinct from all other Asian and European pig breeds. The type II haplotype most likely represents a recent introgression from other Asian pigs. The evolutionary relationships and the population genetic structure of Lanyu pigs revealed here should provide significant information for further conservation of this unusual breed.

\section{Acknowledgements}

This work was supported by a grant from the National Science Council of Taiwan, Republic of China (NSC 922313-B-002-145 and NSC 94-2317-B-002-020) and Council of Agriculture of Taiwan, Executive Yuan, Republic of China [93AD-4.1.3-AS -U1 (4)]. We thank Dr Harry Wilson for editing the manuscript.

\section{References}

Bollen P.J.A., Hansen A.K. \& Rasmussen H.J. (2000) The laboratory swine. In: Important Biological Features (Ed. by M.A. Suckow), pp. 6-9. CRC Press, New York.
Bradley D.G., MacHugh E.E., Cunningham P. \& Loftus R.T. (1996) Mitochondrial diversity and origin of African and European cattle. Proceedings of the National Academy of Sciences of the United States of America 93, 5131-5.

Cheng P. (1986) Pig breeds in China. In: Description of Chinese Pig Breeds (Ed. by Z. Zhang), pp. 150-5. Shanghai Scientific and Technical Publishers, Shanghai.

Dawson A.G. (1992) Ice Age Earth: Late Quaternary Geology and Climate. Chapman and Hall, New York.

FAO (Food and Agriculture Organization of the United Nations) (2004) Secondary Guidelines for Development of National Farm Animal Genetic Resources Management Plans. Measurement of Domestic Animal Diversity (MoDAD): Recommended Microsatellite Markers. http://dad.fao.org/ (accessed on 6 April 2005).

Felsenstein J. (1993) PHYLIP 3.6: Phylogeny Inference Package. University of Washington, Seattle, WA.

Felsenstein J. (2006) PHYLIP Version 3.66 Executables for PowerMac. University of Washington, Seattle, WA.

Giuffra E., Kijas J.M.H., Amarger V., Carlborg O., Jeon J.T. \& Andersson L. (2000) The origin of the domestic pig: independent domestication and subsequent introgression. Genetics 154, 1785-91.

Gongora J., Fleming P., Spencer P.B.S., Mason R., Garkavenko O., Meyer J.N., Droegemueller C., Lee J.H. \& Moran C. (2004) Phylogenetic relationships of Australian and New Zealand feral pigs assessed by mitochondrial control region sequence and nuclear GPIP genotype. Molecular Phylogenetics and Evolution 33, 339-48.

Hein J. \& Stovlbaek J. (1996) Combined DNA and protein alignment. Methods in Enzymology 266, 402-18.

Kim K.I., Lee J.H., Li K., Zhang Y.P., Lee S.S., Gongora J. \& Moran C. (2002) Phylogenetic relationships of Asian and European pig breeds determined by mitochondrial DNA D-loop sequence polymorphism. Animal Genetics 33, 19-25.

Okumura N., Ishiguro N., Nakano M., Hirai K., Matsui A. \& Sahara M. (1996) Geographic population structure and sequence divergence in the mitochondrial DNA control region of the Japanese wild boar (Sus scrofa leucomystax), with reference to those of domestic pigs. Biochemical Genetics 34, 179-89.

Posada D. \& Crandall K.A. (1998) модеLтEST: testing the model of DNA substitution. Bioinformatics 14, 817-18.

Rozas J., Sánchez-Delbarrio J.C., Messeguer X. \& Rozas R. (2003) DNASP DNA polymorphism analyses by the coalescent and other methods. Bioinformatics 19, 2496-7.

Saunders M.A. \& Edwards S.V. (2000) Dynamics and phylogenetic implications of mtDNA control region sequences in New World Jays (Aves: Corvidae). Journal of Molecular Evolution 51, 97-109.

Schmidt H.A., Strimmer K., Vingron M. \& von Haeseler A. (2002) TREE-PUZZLE: maximum likelihood phylogenetic analysis using quartets and parallel computing. Bioinformatics 18, 502-4.

Strimmer K. \& von Haeseler A. (1996) QUARTET PUZZLING: a quartet maximum likelihood method for reconstructing tree topologies. Molecular Biology and Evolution 13, 964-9.

Su B., Xiao J., Underhill P. et al. (1999) Y-chromosome evidence for a northward migration of modern humans into East Asia during the last ice age. American Journal of Human Genetics $\mathbf{6 5}$, 1718-24. 
Su B., Jin L., Underhill P. et al. (2000) Polynesian origins: insights from the Y chromosome. Proceedings of the National Academy of Sciences of the United States of America 97, 8225-8.

Swofford D.L. (2002) PAUP*: Phylogenetic Analysis Using Parsimony (*and other Methods), version 4.0 beta10. Sinauer Associates, Inc., Sunderland, MA.

Wan Q.H., Wu H., Fujihara T. \& Fang S.G. (2004) Which genetic markers for which conservation genetics issues? Electrophoresis 25, 2165-76.

Watanobe T., Okumura N., Ishiguro N., Nakano M., Matsui A., Sahara M. \& Komatsu M. (1999) Genetic relationship and distribution of the Japanese wild boar (Sus scrofa leucomystax) and Ryukyu wild boar (Sus scrofa riukiuanus) analysed by mitochondrial DNA. Molecular Ecology 8, 1509-12.

Watanobe T., Ishiguro N., Nakano M., Takamiya H., Matsui A. \& Hongo H. (2002) Prehistoric introduction of domestic pigs onto the Okinawa Islands: ancient mitochondrial DNA evidence. Journal of Molecular Evolution 55, 222-31.

Watanobe T., Ishiguro N. \& Nakano M. (2003) Phylogeography and population structure of the Japanese wild boar Sus scrofa leucomystax: mitochondrial DNA variation. Zoological Science 20, 1477-89.

\section{Supplementary Material}

The following supplementary material is available for this article online from http://www.blackwell-synergy.com/doi/ full/10.1111/j.1365-2052.2007.01646.x.

Figure S1 Nucleotide sequence differences of the mtDNA control region between type I and II Lanyu pigs.

Figure S2 Variable sites of mtDNA control region sequences of Lanyu pigs, Taoyuan pigs, Australian and New Zealand feral pigs of Asian type, and Asian and European wild and domestic pigs.

Please note: Blackwell Publishing is not responsible for the content or functionality of any supplementary materials supplied by the authors. 